\title{
REGULATION OF ION DRIFTS AND ANISOTROPIES BY PARAMETRICALLY UNSTABLE FINITE-AMPLITUDE ALFVÉN-CYCLOTRON WAVES IN THE FAST SOLAR WIND
}

\author{
Y. G. Maneva ${ }^{1,2,5}$, J. A. Araneda ${ }^{3}$, And E. Marsch ${ }^{4,5}$ \\ ${ }^{1}$ NASA Goddard Space Flight Center, Greenbelt, MD 20771, USA; yana.g.maneva@ nasa.gov \\ ${ }^{2}$ Department of Physics, Catholic University of America, Washington, DC 20064, USA \\ ${ }^{3}$ Departamento de Física, Universidad de Concepción, 4070386, Chile \\ ${ }^{4}$ Institute for Experimental and Applied Physics, Christian Albrechts University at Kiel, D-24118 Kiel, Germany \\ Received 2013 September 25; accepted 2014 January 17; published 2014 February 24
}

\begin{abstract}
We study the preferential heating and differential acceleration of minor ions by dissipation of ion-acoustic waves (IAWs) generated by parametric instabilities of a finite-amplitude monochromatic Alfvén-cyclotron pump wave. We consider the associated kinetic effects of Landau damping and nonlinear pitch-angle scattering of protons and $\alpha$ particles in the tenuous plasma of coronal holes and the fast solar wind. Various data collected by Wind spacecraft show signatures for a local transverse heating of the minor ions, presumably by Alfvén-cyclotron wave dissipation, and an unexpected parallel heating by a so far unknown mechanism. Here, we present the results from a set of 1.5 dimensional hybrid simulations in search for a plausible explanation for the observed field-aligned kinetic features in the fast solar wind minor ions. We investigate the origin and regulation of ion relative drifts and temperature anisotropies in low plasma $\beta$, fast solar wind conditions. Depending on their initial drifts, both ion species can heat up not only transversely through cyclotron resonance and non-resonant wave-particle interactions, but also strongly in the parallel direction by Landau damping of the daughter IAWs. We discuss the dependence of the relative ion drifts and temperature anisotropies on the plasma $\beta$ of the individual species and we describe the effect of the pump wave amplitude on the ion heating and acceleration.
\end{abstract}

Key words: acceleration of particles - instabilities - plasmas - solar wind - turbulence - waves

Online-only material: color figures

\section{INTRODUCTION}

The protons and heavier ions escaping from the Sun are key probes of the underlying heating and acceleration mechanisms operating at the characteristic ion scales in the solar corona and the solar wind. As the solar wind plasma is so tenuous, the rate of Coulomb collisions is generally too low to establish local thermodynamic equilibrium (LTE). Consequently, the velocity distribution functions (VDFs) of each particle species are prone to deviate from an isotropic Maxwellian form. These departures manifest themselves through persistent non-thermal features and affect the different particle species selectively. In fact, decades of in situ measurements show that the preferential heating of minor ions is an intrinsic feature of coronal holes and the fast solar wind, in which $\alpha$ particles and heavy ions are found to be much hotter than the most abundant protons and electrons (Marsch 2006; Cohen et al. 1996; Kohl et al. 2006). Likewise, all species there exhibit anisotropic VDFs, with different temperatures along and across the orientation of the ambient magnetic field.

The nature and magnitude of the ion anisotropy in the solar wind strongly vary with heliocentric distance or, relatedly, with the value of the plasma $\beta$. Thus, in the fast solar wind close to the Sun (at Helios perihelion near 0.29 AU), the core of the protons shows a higher temperature in the perpendicular direction. For the range of Helios measurements between 0.3 and $0.54 \mathrm{AU}$ (Marsch et al. 1982b; Matteini et al. 2007; Bourouaine et al. 2010), the typical values of the proton core anisotropy $T_{p \perp} / T_{p \|}$ range between 2 and 3. The same direction of the anisotropy is often observed for the $\alpha$ particles, but at somewhat lower

\footnotetext{
5 Previously at Max-Planck-Institut für Sonnensystemforschung, 37191 Katlenburg-Lindau, Germany.
}

values of $T_{\alpha \perp} / T_{\alpha \|}$ between 1.2 and 1.5 in the low $\beta$, fast solar wind close to the Sun; see Marsch et al. (1982a). More generally, the temperature anisotropy strongly depends on the local plasma properties, the type of fluctuations in the turbulent spectra, and the available wave power; see Bourouaine et al. (2011). The observed anisotropies, however, differ from the Chew-Goldberger-Low double adiabatic expansion prediction, implying that some constraints should limit the departures from LTE. Many authors describe the limits for the proton and recently $\alpha$ temperature anisotropy $T_{\perp} / T_{\|}$within the stability thresholds, as derived from the linear kinetic theory of the Vlasov-Maxwell set of equations (Matteini et al. 2007, 2013; Bale et al. 2009; Maruca et al. 2011, 2012). The results from this paper show that nonlinear effects can be very important for the proper treatment of the kinetic plasma instabilities in the solar wind, even in the regime of rather small wave amplitudes.

Another feature, related to the temperature anisotropy, and also suggesting a selective process in the solar wind, is the preferential acceleration of $\alpha$ particles and other minor ion species. The drift speed of $\alpha$ particles $V_{\alpha p}$ relative to the main proton population is observed to be a sizable fraction of the local Alfvén speed $V_{\mathrm{A}}$ and, apart from the ion densities and the plasma $\beta$, the ion anisotropies depend also on the differential streaming between the constituent ion species. Wind measurements made near 1 AU (Kasper et al. 2008, 2013; Maruca et al. 2012) indicate that fast solar wind protons possess a predominantly parallel temperature anisotropy for all values of the relative drift speed, whereas the heating of the $\alpha$ particles varies significantly with $V_{\alpha p}$. Thus, they exhibit high perpendicular anisotropies $\left(T_{\alpha \perp} / T_{\alpha \|}>1\right)$ when the two species move at similar speeds, but higher parallel temperatures for relative drifts $V_{\alpha p} / V_{\mathrm{A}}$ in the range from 0.15 to 0.85 . 
One of the numerous attempts to explain the observed ion features in the turbulent fast solar wind invokes wave-particle interactions with resonant ion-cyclotron waves (ICWs). For a long time, the scientific community has faced the question of whether ICWs exist in the solar corona and whether they can be responsible for the observed ion heating and acceleration in the solar wind (Hollweg \& Isenberg 2002; Marsch 2006). However, the detection of small-scale magnetic waves and perturbations in the solar corona still remains a challenge even with modern remote-sensing techniques. There exists indirect evidence implying the presence of Alfvén waves in the solar corona (Jess et al. 2009; Banerjee et al. 2009; Tomczyk et al. 2007), but currently there are no means to measure high-frequency plasma waves near the ion-cyclotron resonance there. Only recently have parallel and quasi-parallel ICWs been unambiguously detected by STEREO in the fast solar wind near 1 AU (Jian et al. 2009) and by Messenger near 0.3 AU (Jian et al. 2010). These waves have finite amplitudes (constituting few percent of the magnitude of the background magnetic field) and are expected to become even stronger as one gets closer to the Sun. Evidence based on Helios data for ICWs, with increasing power spectral density, which lies far beyond the linear-theory range at shorter heliocentric distances, was recently presented in Bourouaine et al. (2011).

On the other hand, low-frequency, nonlinear Alfvén waves have long been observed ubiquitously in the fast solar wind over a wide range of solar distances (Tu \& Marsch 1995; Bruno \& Carbone 2005). In addition, finite-amplitude Alfvén-cyclotron waves have long been theoretically known as exact solutions of the hot plasma kinetic equation (Sonnerup \& Su 1967; AbrahamShrauner \& Feldman 1977) and numerical simulations have recently shown that parametric instabilities of those waves can lead to preferential heating and acceleration of minor ions under low plasma $\beta$ conditions (Araneda et al. 2009; Maneva et al. $2009,2010,2013 b$ ), as prevailing in the solar corona.

In this paper, we present numerical results from a parametric simulation study of the regulation of the ion drifts and temperature anisotropies via parametrically unstable Alfvén-cyclotron waves and give an explanation of the enhanced parallel heating of $\alpha$ particles for a large range of their relative drifts. We investigate the dependence of ion heating and acceleration for a variety of different initial relative drifts between the two ion species and for different initial parallel plasma $\beta$ conditions, in order to draw conclusions about how the heating and acceleration mechanisms could possibly operate in the fast solar wind. Furthermore, we comment on the influence the finite-amplitude pump wave has on the regulation of the relative drifts and ion temperature anisotropies - a purely nonlinear effect, which cannot be described within the scope of the linear Vlasov theory.

We should note that the initial pump wave we choose is stable with respect to linear instability analysis and only becomes unstable once nonlinear wave-wave couplings, such as parametric instabilities, start to operate. Parametric instabilities are common features of both warm and cold plasmas, where finiteamplitude waves are present. During the instability process, the pump wave couples to the thermal motion of the plasma, generating a daughter electromagnetic wave and feeding energy into a daughter sound (ion-acoustic) wave. For the original work on fluid-type parametric instabilities with streaming $\alpha$ particles, we refer the reader to Hollweg et al. (1993) and for kinetic effects within the same setup, we refer the reader to Kauffmann \& Araneda (2008). Although the three-wave processes occur instantly in the very early stage of the simulations, it typically takes a few hundred proton gyroperiods $\left(300-500 \Omega_{p} t\right)$ for a fully nonlinear stage to develop, with the parametric decay of the pump and a generation of broad turbulent spectra of daughter ion-acoustic and Alfvén-cyclotron waves. At that stage, the pump is no longer dominant and the dynamics of the system are purely driven by the existing turbulence.

\section{NUMERICAL TECHNIQUES}

We performed a series of 1.5 dimensional (1.5D) hybrid simulations to study the behavior of the relative ion drifts and anisotropies, which originate from the nonlinear evolution of initially isotropic ion velocity distributions in a collisionless homogeneous plasma that is permeated by a parametrically unstable finite-amplitude Alfvén-cyclotron pump wave. The code is 1D in space, but retains all three components of the electromagnetic fields and the ion velocities. It treats the electrons as a warm isothermal and massless fluid and fully resolves the ions kinetically. We do not consider the dynamics of the electrons, but they contribute to the electromagnetic fields to ensure that quasi-neutrality on ion scales is maintained.

For the ions, we use a standard particle-in-cell method to solve the coupled set of the Maxwell-Vlasov equations (Winske \& Leroy 1984; Terasawa et al. 1986). Particle positions and velocities are advanced in time with a leap-frog algorithm, whereas the fields are time advanced with an explicit method and their spatial derivatives are calculated with a fourth-order finite-difference scheme. All calculations are performed in the plasma frame of reference where the total momentum vanishes and the background magnetic field is oriented in the $z$-direction, $\mathbf{B}_{0}=B_{0} \hat{z}$.

We initialize the ion simulations with isotropic Maxwellian VDFs, differentially streaming along the background magnetic field. In addition, a coherent perpendicular motion is imposed on the ions (see Maneva et al. 2010), self-consistently due to the presence of the pump wave (propagating parallel to the magnetic field) with a frequency below the $\alpha$ particle resonance frequency $\omega_{0}<\Omega_{\alpha}$. The simulation box has 2048 cells and a length corresponding to 32 times the wavelength of the pump wave $2 \pi / k_{0}$. The spatial resolution is chosen to address both short- and long-wavelength instabilities simultaneously and the integration time step is set to $\Omega_{p} \Delta t=0.035$. In each simulation run, we use 1000 particles per cell for each ion species and we assume periodic boundary conditions for calculating both the positions of the particles and the electromagnetic fields. The plasma $\beta$ for the electrons is fixed to the value $\beta_{e} \equiv 2 \mu_{0} n_{e} k_{\mathrm{B}} T_{e} / B_{0}^{2}=0.5$, whereas the ions have a variable plasma $\beta$ defined by $\beta_{i}=n_{i} k_{B} T_{i} /\left(B_{0}^{2} / 2 \mu_{0}\right)$. The number density of the $\alpha$ particles is fixed to $5 \%$ of the electron number density, $n_{\alpha}=0.05 n_{e}$, and the Alfvén speed is defined as $V_{\mathrm{A}}=B_{0} /\left(\mu_{0} n_{e} m_{p}\right)^{1 / 2}$.

The simulations were performed for four different initial values of the plasma $\beta_{i}$-related dimensionless measure of the ion temperature, as given by the square of the ratio of the thermal to Alfvén speed: $\tilde{\beta}_{i}=\left(2 k_{B} T_{i} / m_{i}\right) / V_{\mathrm{A}}{ }^{2}$. For each initial $\tilde{\beta}_{i}$, in addition, we selected a range of different initial values for the differential streaming between the two ion species $V_{\alpha p}$, starting with non-drifting particles and then increasing the values of the initial drift speed at a step size of 5\% of the local Alfvén speed.

The initial monochromatic pump wave represents an exact nonlinear solution of the collisionless hot plasma kinetic equation (Sonnerup \& Su 1967) for a parallel-propagating, left-hand, circularly polarized wave and is self-consistently coupled to the 
motion of the initially isotropic and uniform density plasma, as described in Araneda et al. (2009) and Maneva et al. (2013b). In the non-relativistic case and for wave frequencies much lower that the electron gyration $\left(\omega_{0} \ll \Omega_{e}\right)$, the dispersion relation for the pump wave is given by

$$
k_{0}^{2}-\frac{\mu_{0}}{B_{0}^{2}} \sum_{i \neq e} n_{i} m_{i} \frac{\left(\omega_{0}-V_{i} k_{0}\right)^{2}}{1-\omega_{0} / \Omega_{i}+V_{i} k_{0} / \Omega_{i}}=0,
$$

where $V_{i}$ is the bulk drift speed for each species in the center of mass frame, which allows a self-consistent initial ion differential streaming, $V_{\alpha p}=V_{\alpha}-V_{p}$. Note that Equation (1) has the same form as the cold plasma dispersion relation used for linear stability studies or to initialize a wave spectrum in hybrid simulations. We should note that in the context of the present paper, the meanings of $\omega_{0}$ and $k_{0}$ differ from the linear theory consideration as we are considering finite-amplitude waves. We set the value of the pump wave number to $k_{0}=0.4 \Omega_{p} / V_{\mathrm{A}}$, so that for each value of the initial differential speed $V_{\alpha p}$ we have a slightly different pump wave frequency. Typically, it amounts to about $30 \%$ of the proton gyrofrequency $\left(\omega_{0} \approx 0.3 \Omega_{p}\right.$; Table 1$)$, a frequency corresponding to that of the majority of the ICWs recently observed by STEREO (Jian et al. 2009).

For the initial state, we choose a single pump mode, which lies on the lower branch of the left-hand polarized Alfvén-cyclotron of Equation (1). It corresponds to fluctuations in the transverse direction, $\delta \mathbf{B}=\delta B\left[\cos \left(k_{0} z-\omega_{0} t\right) \hat{\mathbf{x}}+\sin \left(k_{0} z-\omega_{0} t\right) \hat{\mathbf{y}}\right]$. The selfconsistent bulk velocities of the ions in the transverse direction are correspondingly given by

$$
\mathbf{V}_{\perp i}=-\frac{\left(\omega_{0} / k_{0}-V_{i}\right)}{1-\omega_{0} / \Omega_{i}+k_{0} V_{i} / \Omega_{i}} \delta \mathbf{B} .
$$

A similar setup was implemented by Nariyuki et al. (2009) in their study of parametric instabilities of circularly polarized waves in a proton/proton plasma.

The relative amplitude with respect to the magnitude of the external magnetic field $\delta B / B_{0}$ was chosen to be in the range of $0.15-0.25$. Regarding the wave amplitude, we should note once again that finite-amplitude nonlinear Alfvén waves are ubiquitously detected in the high-speed streams at different heliocentric distances (Tu \& Marsch 1995; Bruno \& Carbone 2005) and that there are now strong indications for the existence of high-frequency Alfvén ICWs as well (Jian et al. 2009, 2010; Bourouaine et al. 2011). Nevertheless, we shall discuss more about the effect of a decreasing pump amplitude below.

\section{RESULTS AND DISCUSSION}

In order to investigate the dependence of the relative drifts on the ion anisotropies and their relation to the wave spectra at different heliospheric distances from the Sun, we perform a series of $1.5 \mathrm{D}$ hybrid simulations, compare the results to in situ solar wind observations, and comment on the relevant ion heating and acceleration mechanisms. Plausible ways to understand the observed ion relative drifts and anisotropies in the fast solar wind have been explored earlier by several authors (Hellinger et al. 2005; Gary et al. 2006; Nariyuki et al. 2009; Araneda et al. 2009; Verscharen \& Chandran 2013; Galinsky \& Shevchenko 2012, 2013; Perrone et al. 2013). Here, we aim to broaden and corroborate these studies by investigating further the kinetic behavior of heavier ions, especially in the case when differential streaming and finite-amplitude waves are present in the plasma.

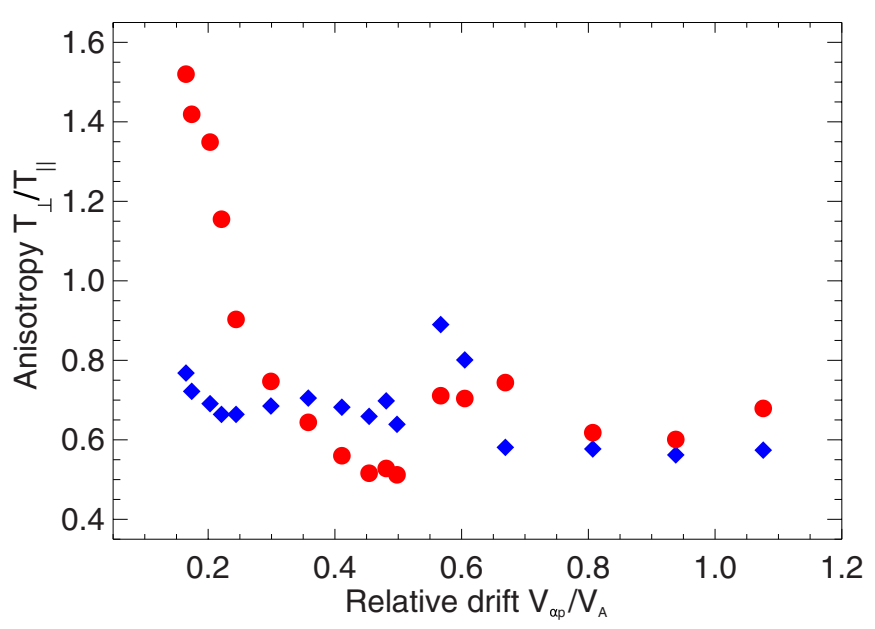

Figure 1. Dependence of the ion temperature anisotropy $T_{\perp} / T_{\|}$on the relative drift speed between protons and $\alpha$ particles, $V_{\alpha p} / V_{\mathrm{A}}$, for $\tilde{\beta}_{\alpha}=0.02$ and $\tilde{\beta}_{p}=0.08$, corresponding to case (a) from Table 1 . Proton anisotropy is plotted as blue rhombuses and $\alpha$ anisotropy is shown as red circles.

(A color version of this figure is available in the online journal.)

The simulations cover four different initial $\tilde{\beta}$ regimes. Additionally, for each value of $\tilde{\beta}$, we have selected a wide range of initial drift speeds (starting with zero drift and slightly increasing the values with a step size of $0.05 V_{\mathrm{A}}$ ), corresponding to different initial pump wave frequencies, as given by the kinetic plasma dispersion relation in Equation (1). The initial setups represent the two typical cases of equal temperatures, $T_{\alpha}=T_{p}$ (with $\tilde{\beta}_{\alpha}=0.02 ; \tilde{\beta}_{p}=4 \tilde{\beta}_{\alpha}=0.08$ ), or equal thermal speeds of the two ion species, in both low- and high-plasma $\beta$ regimes to mimic the conditions in coronal holes $\left(\tilde{\beta}_{\alpha}=\tilde{\beta}_{p}=0.08\right)$ and at different solar radii in the fast solar wind $\left(\tilde{\beta}_{\alpha}=\tilde{\beta}_{p}=0.2\right.$ and $\left.\tilde{\beta}_{\alpha}=\tilde{\beta}_{p}=0.35\right)$. The first case relates to the possible origin of the ion anisotropy and preferential heating for an initially isothermal plasma, whereas the last cases correspond to the typical solar wind ion parameters observed recently at $1 \mathrm{AU}$ (Berger et al. 2010, 2011). A comparison between simulation results and in situ measurements provides important clues for the ion heating and acceleration mechanisms operating in coronal holes and the associated fast solar wind.

The characteristic initial parameters used for the initialization of the respective simulation are presented in Table 1. Along with the pump-wave frequency, the phase speed of the wave and the initial and final ion relative drifts, the table includes the outcome values for the ion temperature ratios, $T_{\| \alpha} / T_{\| p}$ and $T_{\perp \alpha} / T_{\perp p}$, for all $\tilde{\beta}$ cases investigated. Runs marked by a star did not reach a saturated final state. As we aim to investigate the origin of ion temperature anisotropies and relative drift speeds in a low $\beta$ plasma region close to the Sun and possibly relate them to the available data near $1 \mathrm{AU}$, we have excluded the runs with non-stationary final stages from the temperature ratios and temperature anisotropy plots presented below in Figures 1-8. Even though the final stages of those runs do not reach a dynamic wave-particle-interaction equilibrium, some of them (with initial drifts close to the local Alfvén speed, exceeding the value of the phase speed of the pump wave; Table 1) show a quasi-stable initial state, which lasted for about 300 proton gyroperiods. During that early stage, the ion temperatures and drifts remain stable, until the thermal noise in the system (always present due to the random motion of the particles) increases 
Table 1

Parameters for the Different Numerical Simulation Runs

\begin{tabular}{|c|c|c|c|c|c|c|c|c|c|c|c|c|c|}
\hline Run No. & $\omega_{0}$ & $\omega_{0} / k_{0}$ & $V_{\alpha p, \text { in }}$ & $V_{\alpha}$ & $V_{p}$ & $V_{\alpha p, \text { fin }}^{(\mathrm{a})}$ & $V_{\alpha p, \text { fin }}^{(\mathrm{b})}$ & $V_{\alpha p, \text { fin }}^{(\mathrm{c})}$ & $V_{\alpha p, \text { fin }}^{(\mathrm{d})}$ & $R_{\perp}^{\text {(a) }}$ & $R_{\|}^{(\mathrm{a})}$ & $R_{\perp}^{(\mathrm{b})}$ & 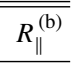 \\
\hline 1 & 0.2995 & 0.749 & 0 & 0 & 0 & 0.165 & 0.143 & 0.089 & 0.095 & 12.66 & 6.40 & 14.23 & 6.27 \\
\hline 2 & 0.3026 & 0.757 & 0.05 & 0.041 & -0.009 & 0.174 & 0.178 & 0.115 & 0.131 & 10.46 & 5.32 & 12.13 & 5.35 \\
\hline 3 & 0.3049 & 0.762 & 0.1 & 0.082 & -0.018 & 0.203 & 0.202 & 0.156 & 0.167 & 8.59 & 4.40 & 10.08 & 4.86 \\
\hline 4 & 0.3064 & 0.766 & 0.15 & 0.123 & -0.027 & 0.221 & 0.240 & 0.190 & 0.206 & 6.41 & 3.68 & 8.61 & 4.65 \\
\hline 5 & 0.3072 & 0.768 & 0.2 & 0.164 & -0.036 & 0.244 & 0.292 & 0.217 & 0.232 & 4.85 & 3.57 & 7.56 & 4.69 \\
\hline 6 & 0.3074 & 0.769 & 0.25 & 0.205 & -0.045 & 0.299 & 0.338 & 0.251 & 0.277 & 4.10 & 3.76 & 6.63 & 4.64 \\
\hline 7 & 0.3070 & 0.768 & 0.3 & 0.245 & -0.055 & 0.358 & 0.388 & 0.281 & 0.325 & 3.59 & 3.93 & 5.95 & 4.71 \\
\hline 8 & 0.3062 & 0.766 & 0.35 & 0.286 & -0.064 & 0.411 & 0.428 & $0.307^{*}$ & $0.344^{*}$ & 3.22 & 3.93 & 5.33 & 4.59 \\
\hline 9 & 0.3049 & 0.762 & 0.4 & 0.327 & -0.073 & 0.454 & 0.462 & $0.338^{*}$ & $0.385^{*}$ & 2.97 & 3.80 & 4.90 & 4.27 \\
\hline 10 & 0.3032 & 0.758 & 0.45 & 0.368 & -0.082 & 0.481 & 0.491 & $0.370^{*}$ & $0.404^{*}$ & 2.79 & 3.69 & 4.49 & 4.02 \\
\hline 11 & 0.3012 & 0.753 & 0.5 & 0.409 & -0.091 & 0.498 & 0.500 & $\ldots$ & $\ldots$ & 2.72 & 3.39 & 4.27 & 3.70 \\
\hline 12 & 0.2988 & 0.747 & 0.55 & 0.450 & -0.100 & $0.472^{*}$ & $0.498^{*}$ & $\ldots$ & $\ldots$ & $3.04^{*}$ & $3.02^{*}$ & $4.11^{*}$ & $3.49^{*}$ \\
\hline 13 & 0.2963 & 0.741 & 0.6 & 0.491 & -0.109 & $0.420^{*}$ & $0.496^{*}$ & $\ldots$ & $\ldots$ & $3.19^{*}$ & $2.84^{*}$ & $3.96^{*}$ & $3.39^{*}$ \\
\hline 14 & 0.2904 & 0.726 & 0.7 & 0.573 & -0.127 & $0.441^{*}$ & $0.489^{*}$ & $\ldots$ & $\ldots$ & $2.25^{*}$ & $3.03^{*}$ & $3.54^{*}$ & $3.61^{*}$ \\
\hline 15 & 0.2837 & 0.709 & 0.8 & 0.655 & -0.145 & $0.480^{*}$ & $0.546^{*}$ & $\ldots$ & $\ldots$ & $2.22^{*}$ & $3.24^{*}$ & $3.22^{*}$ & $3.96^{*}$ \\
\hline 16 & 0.2765 & 0.691 & 0.9 & 0.736 & -0.164 & $0.548^{*}$ & $0.588^{*}$ & $\ldots$ & $\ldots$ & $2.27^{*}$ & $3.54^{*}$ & $3.73^{*}$ & $4.02^{*}$ \\
\hline 17 & 0.2688 & 0.672 & 1.0 & 0.818 & -0.181 & $0.601^{*}$ & $0.671^{*}$ & $\ldots$ & $\ldots$ & $3.17^{*}$ & $3.29^{*}$ & $4.17^{*}$ & $4.11^{*}$ \\
\hline 18 & 0.2606 & 0.652 & 1.1 & 0.900 & -0.200 & $0.721^{*}$ & $0.725^{*}$ & $\ldots$ & $\ldots$ & $4.40^{*}$ & $2.92^{*}$ & $5.49^{*}$ & $3.93^{*}$ \\
\hline 19 & 0.2521 & 0.630 & 1.2 & 0.982 & -0.218 & $0.653^{*}$ & $0.716^{*}$ & $\ldots$ & $\ldots$ & $6.71^{*}$ & $3.42 *$ & $7.43^{*}$ & $4.19^{*}$ \\
\hline 20 & 0.2433 & 0.608 & 1.3 & 1.064 & -0.236 & $0.641^{*}$ & $0.701^{*}$ & $\ldots$ & $\ldots$ & $8.54^{*}$ & $4.23^{*}$ & $9.05^{*}$ & $5.43^{*}$ \\
\hline 21 & 0.2342 & 0.586 & 1.4 & 1.145 & -0.255 & $0.620^{*}$ & $0.702^{*}$ & $\ldots$ & $\ldots$ & $9.29^{*}$ & $5.0 * 9$ & $0.75^{*}$ & $6.09 *$ \\
\hline 22 & 0.2249 & 0.562 & 1.5 & 1.227 & -0.273 & 0.669 & $0.869^{*}$ & $\ldots$ & $\ldots$ & 8.01 & 6.25 & $10.63^{*}$ & $6.57^{*}$ \\
\hline 23 & 0.2154 & 0.539 & 1.6 & 1.309 & -0.291 & 0.807 & $1.109^{*}$ & $\ldots$ & $\ldots$ & 7.48 & 6.99 & $9.86^{*}$ & $6.31^{*}$ \\
\hline 24 & 0.2057 & 0.514 & 1.7 & 1.391 & -0.309 & 1.076 & $1.216^{*}$ & $\ldots$ & $\ldots$ & 7.82 & 6.61 & $10.38^{*}$ & $6.63^{*}$ \\
\hline 25 & 0.1958 & 0.489 & 1.8 & 1.473 & -0.327 & 1.333 & $1.434^{*}$ & $\ldots$ & $\ldots$ & 7.60 & 6.16 & $9.54^{*}$ & $6.09^{*}$ \\
\hline
\end{tabular}

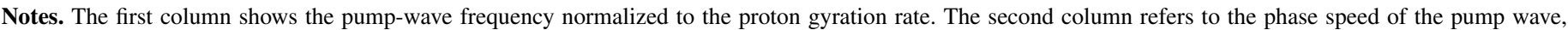

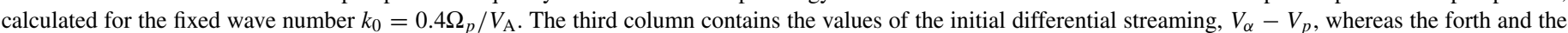

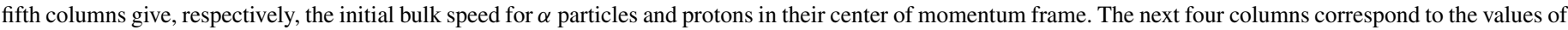

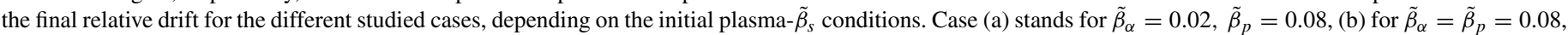

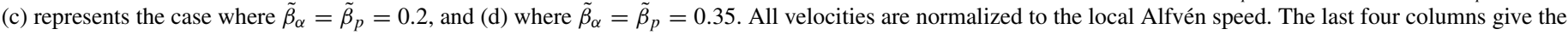

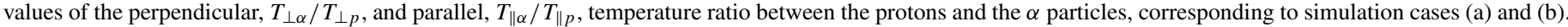

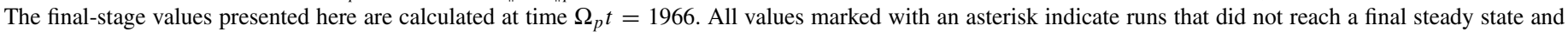
therefore have been excluded from the simulation plots given in the various figures.

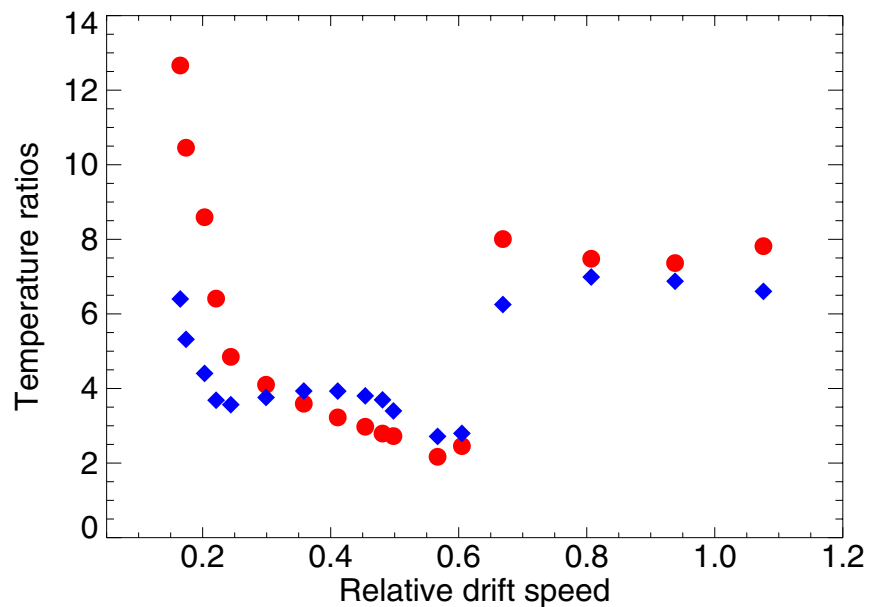

Figure 2. Dependence of the parallel (blue rhombuses) and perpendicular (red circles) temperature ratios of protons and $\alpha$ particles on the normalized relative drift between these two species. All the data points relate to the final stages of the simulations, when a stationary state was reached. The initial conditions correspond to case (a) from Table 1 with $\tilde{\beta}_{\alpha}=0.02$ and $\tilde{\beta}_{p}=0.08$.

(A color version of this figure is available in the online journal.)

to a value sufficient to initiate parametric instabilities. They then lead to the decay of the pump, so that it could no longer maintain stability at those high values of the initial relative drift, with $V_{\alpha p} \geqslant V_{\mathrm{A}}$. As the pump wave depletes, its constraints on

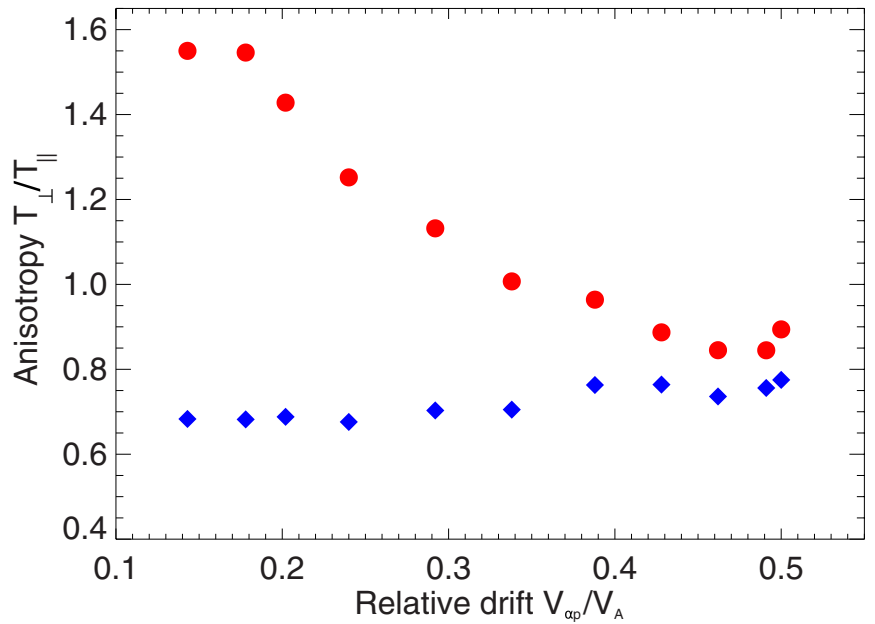

Figure 3. Dependence of the ion temperature anisotropy $T_{\perp} / T_{\|}$on the relative drift speed between protons and $\alpha$ particles, $V_{\alpha p} / V_{\mathrm{A}}$, for $\tilde{\beta}_{\alpha}=\tilde{\beta}_{p}=0.08$, corresponding to case (b) from Table 1. Red circles stand for $\alpha$ s and blue rhombuses stand for protons.

(A color version of this figure is available in the online journal.)

the perpendicular ion motion diminish; see Equation (2). What follows is a diffusion in phase space. Simultaneously, a range of acoustic waves is produced, with a consequent reduction of the differential streaming. We should note that for all the values 


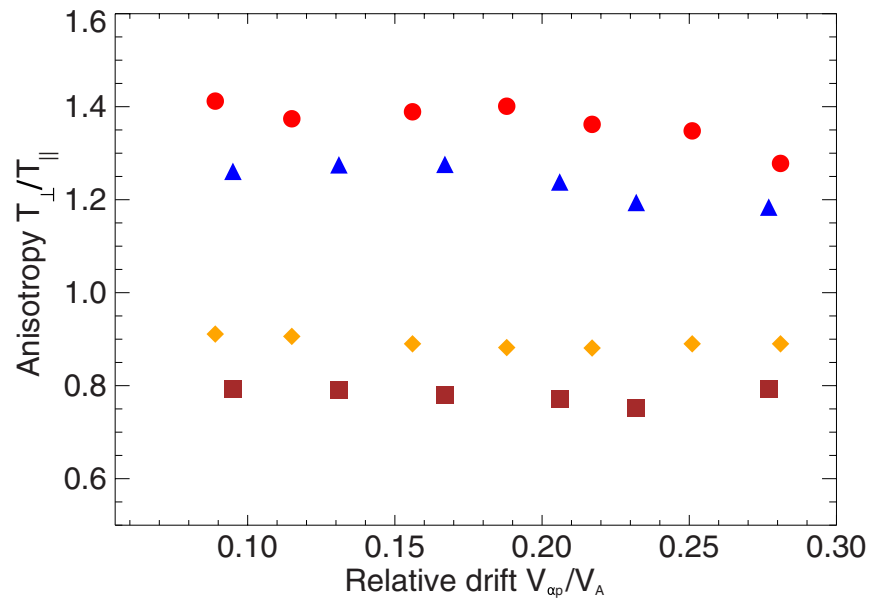

Figure 4. Combined plot of the final ion temperature anisotropy, $T_{\perp} / T_{\|}$, vs the final differential speed, $V_{\alpha p \text {, fin }}$ for cases (c) and (d) from Table 1. The blue triangular and brown square data points correspond to the initial value $\tilde{\beta}_{\alpha}=\tilde{\beta}_{p}=0.2$, whereas the red circles and orange rhombuses denote the case with $\tilde{\beta}_{\alpha}=\tilde{\beta}_{p}=0.35$. The differential speed is normalized to the constant Alfvén speed $V_{\mathrm{A}}$, set by the homogeneous background magnetic field, the proton mass, and the electron number density.

(A color version of this figure is available in the online journal.)

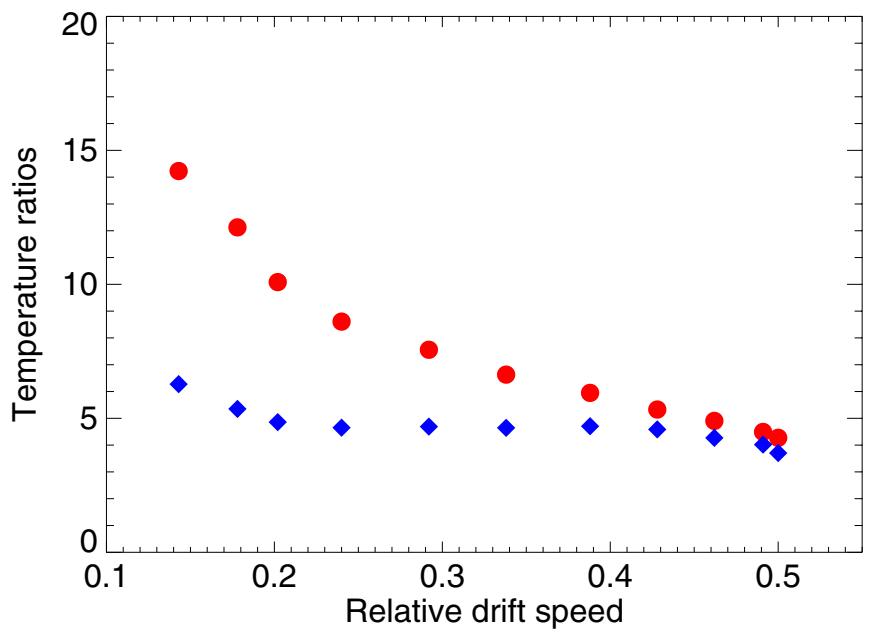

Figure 5. Same relation as shown on Figure 2, but for case (b), initialized with $\tilde{\beta}_{\alpha}=\tilde{\beta}_{p}=0.08$. $\alpha$ s are shown in red circles and protons are plotted with blue rhombuses.

(A color version of this figure is available in the online journal.)

of the relative drift speed considered here, the system is stable with respect to the linear Vlasov instability analysis and the decrease of the relative drifts for $V_{\alpha p}>0.5 V_{\mathrm{A}}$ and the associated generation of IAWs is entirely a nonlinear process, which can be captured well by the kinetic parametric instabilities theory. We should stress that this fully nonlinear effect is present for arbitrary small pump-wave amplitudes and does not disappear as we decrease the strength of the pump wave.

In order to investigate the regulation of the ion anisotropies with increasing initial drifts, we will concentrate here on the bulk moments as computed at the final stages of the simulations. Due to the large number of free parameters, a complete description of the parametric instabilities with a detailed view of the underlying kinetic effects is beyond the scope of the current study and will be presented elsewhere.

Figure 1 shows results from an ensemble of simulations for the ion anisotropies versus relative drift speed $V_{\alpha p}$ for simulation

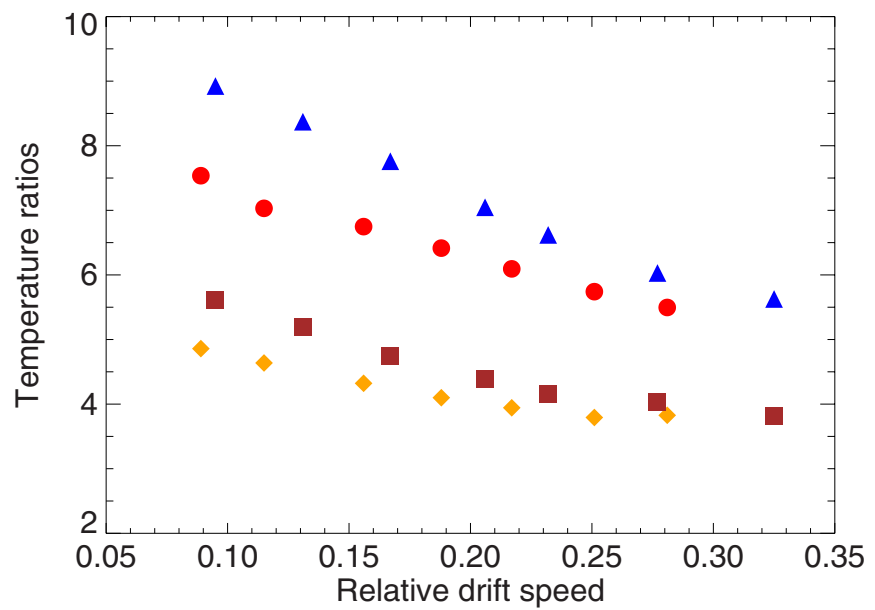

Figure 6. Dependence of the temperature ratios between protons and $\alpha$ particles on the relative drift for cases (c) and (d). The blue and brown curves denote, respectively, the perpendicular and parallel temperature ratios for initial values $\tilde{\beta}_{\alpha}=\tilde{\beta}_{p}=0.2$. The red and orange data points refer to $\tilde{\beta}_{\alpha}=\tilde{\beta}_{p}=0.35$.

(A color version of this figure is available in the online journal.)

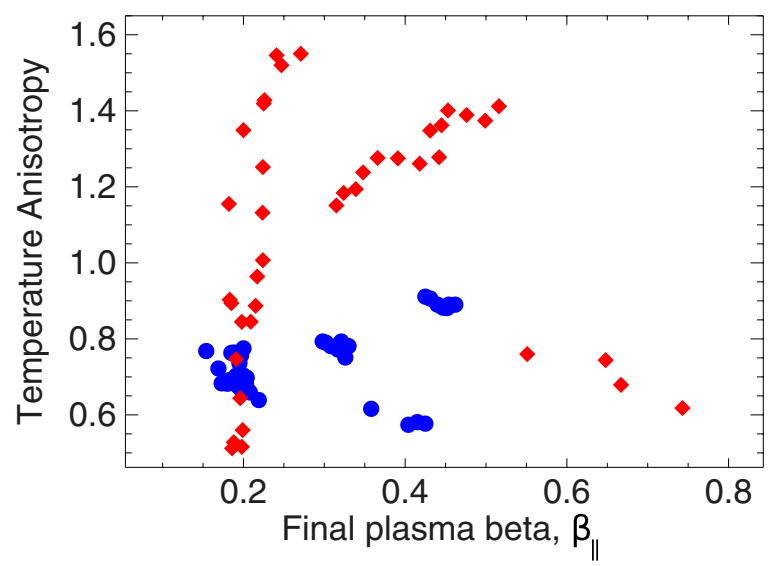

Figure 7. Final temperature anisotropy for the protons (shown by blue circles) and $\alpha$ particles (denoted by red rhombuses) vs. the final parallel $\tilde{\beta}$. The anisotropy points for the $\alpha$ particles are much more scattered, whereas the anisotropies for the protons appear to be more confined, with $T_{\perp} / T_{\|}$in a smaller range between 0.55 and 0.95 . In all simulated cases, Landau damping of the daughter IAWs occurred and proton beams were formed concurrently (without simultaneous perpendicular heating), which resulted in the observed parallel anisotropies of the protons.

(A color version of this figure is available in the online journal.)

case (a) with initially equal temperatures for the protons and $\alpha$ particles, $\tilde{\beta}_{\alpha}=0.02$ and $\tilde{\beta}_{p}=0.08$. The dependence of the ion anisotropies on the relative drifts is qualitatively similar to fast solar wind measurements based on Wind data at $1 \mathrm{AU}$ near the Earth (Kasper et al. 2008, 2013; Maruca et al. 2012). The $\alpha$ particles exhibit an enhanced perpendicular heating for low values of the relative drift speed, $V_{\alpha p}<0.2 V_{\mathrm{A}}$, and a prominent parallel heating for higher initial drifts. The proton temperature ratios indicate at all relative drifts a higher parallel temperature, due to the proton beam formation (Araneda et al. 2009; Matteini et al. 2010; Maneva et al. 2013b). For the range of relative drift speeds, $V_{\alpha p} / V_{\mathrm{A}}$ between 0.35 and 0.5 , the $\alpha$ particles are heated very efficiently by IAW absorption and acquire very large temperatures in the parallel direction. This heating results in low values of the temperature anisotropy for the $\alpha$ particles, with the proton anisotropy exceeding that of the $\alpha \mathrm{s}$. 

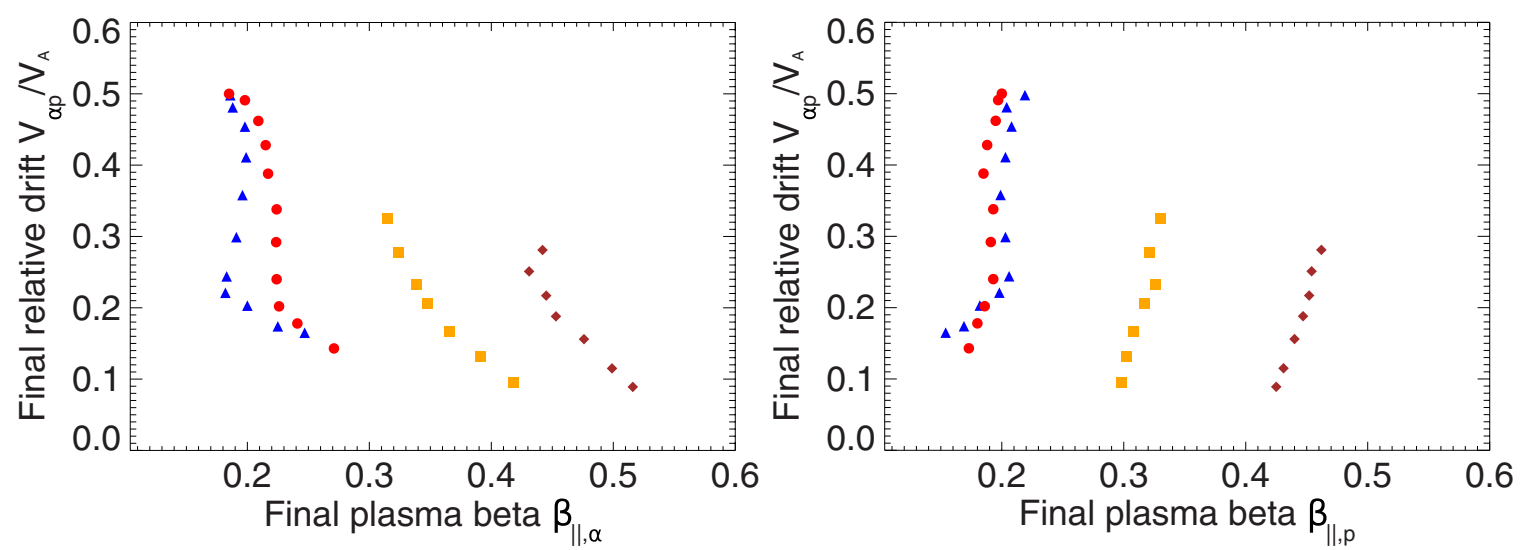

Figure 8. Final normalized ion differential speed vs. final parallel plasma $\tilde{\beta}_{s}$. The left panel refers to the protons and the right one to the $\alpha$ particles. The different colors and symbols correspond to the four investigated initial plasma $\tilde{\beta}$ conditions: $\tilde{\beta}_{\alpha}=0.02, \tilde{\beta}_{p}=0.08$ (blue triangles), $\tilde{\beta}_{\alpha}=\tilde{\beta}_{p}=0.08$ (red circles), $\tilde{\beta}_{\alpha}=\tilde{\beta}_{p}=0.2$ (orange squares), and $\tilde{\beta}_{\alpha}=\tilde{\beta}_{p}=0.35$ (brown rhombuses).

(A color version of this figure is available in the online journal.)

Figure 3 demonstrates the relation between ion drifts and anisotropies in the same format as Figure 1, but for case (b), where the ion species were initialized with equal thermal speeds, $\tilde{\beta}_{\alpha}=\tilde{\beta}_{p}=0.08$. The ion anisotropies show similar behavior and once again resemble the observed features presented in Kasper et al. (2008). This time, fewer data points were included due to the fact that at higher drift speeds the instabilities are stronger and the simulations did not reach a stationary state. We observed that for the saturated cases no regime with $\left(T_{\perp} / T_{\|}\right)_{p}>\left(T_{\perp} / T_{\|}\right)_{\alpha}$ is reached. Again, the protons remain parallely heated at all relative drifts, whereas the $\alpha$ particles at low drifts heat preferentially in the transverse direction and at higher drifts - in parallel. Here, the slope of the temperature anisotropy curve for the $\alpha$ particles is less steep in comparison with the previous case, Figure 1, where the $\alpha$ s are initially slightly colder.

As we increase the plasma $\tilde{\beta}$, it gets more and more difficult to reach a stationary regime with a saturated final wave-particle equilibrium state, even for low values of the relative drift speed (see Table 1). Figure 4 shows how the temperature anisotropies profile changes for a hot plasma in a high- $\tilde{\beta}$ regime. The blue triangles (for $\alpha$ particles) and brown squares (for protons) correspond to case (c) with $\tilde{\beta}_{\alpha}=\tilde{\beta}_{p}=0.2$. The red circles (for $\alpha$ particles) and orange rhomboid curve (for protons) correspond to case (d), where $\tilde{\beta}_{\alpha}=\tilde{\beta}_{p}=0.35$. We can see that the slope of the temperature anisotropy curves becomes almost flat and $\left(T_{\perp} / T_{\|}\right)_{\alpha}$ is no longer steep, as in the case of lower initial $\tilde{\beta}_{s}$, or as observed. The proton temperature anisotropy slightly increases from $\left(T_{\perp} / T_{\|}\right)_{p} \sim 0.7$ in cases (a) and (b) to $\left(T_{\perp} / T_{\|}\right)_{p} \sim 0.8$ and $\left(T_{\perp} / T_{\|}\right)_{p} \sim 0.9$ for cases (c) and (d), respectively. Unlike the protons, the anisotropy for the $\alpha$ particles is not a monotonic function of the plasma $\tilde{\beta}$.

Analyzing these results, we can conclude that if nonlinear Alfvén-cyclotron waves are responsible for the temperature anisotropies and the particles' acceleration, then the observed differential heating should start preferentially in a low $-\tilde{\beta}$ regime, close to the Sun. As we shall see, the simulations also show that for the two investigated cases with a high initial $\tilde{\beta}$, the bulk of the ions cannot be accelerated up to more than a small fraction of the local Alfvén speed, with $V_{\alpha p} / V_{\mathrm{A}}$ in the range of 0.25-0.3. Thus, if one assumes that the observed anisotropies are caused by some local heating mechanism, additionally one needs to think of a way how to differentially accelerate the ions in the first place. On the contrary, in low- $\tilde{\beta}$ conditions, the non-thermal ion heating and acceleration by finite-amplitude Alfvén-cyclotron waves occur simultaneously, as shown previously by Araneda et al. (2009) and Maneva et al. (2013b), which makes it a plausible mechanism likely acting in the coronal holes emitting fast solar wind streams.

The subsequent Figures 2-6 show the parallel and perpendicular temperature ratios between the two ion species, as they appear at the final stages of our simulations for the different initial regimes of $\tilde{\beta}$ considered here, plotted as a function of the final relative drift speed $V_{\alpha p}$, fin $/ V_{\mathrm{A}}$.

Figure 2 describes case (a), where the two ion species are initialized with equal temperatures. In the range of $V_{\alpha p \text {, fin }} / V_{\mathrm{A}}$ from $0.35-0.5$, the perpendicular temperature ratios appear to be particularly reduced, whereas the parallel ones stay almost constant (or slightly increase). This result coincides with the regime in the ion anisotropies in Figure 1, where the anisotropy of the protons exceeds that of the $\alpha$ particles. In that case, the low-temperature anisotropy of the $\alpha$ particles $\left(T_{\perp} / T_{\|}<0.7\right)$ is associated with reduced perpendicular heating. On the contrary, at higher relative drifts $V_{\alpha p \text {, fin }}>0.5 V_{\mathrm{A}}$, the low-temperature anisotropy is related to a preferential parallel heating of the $\alpha$ particles. We should note that the above dependence of the temperature ratios on the ion relative drifts differs from the values presented by Kasper et al. (2008).

Figure 5 shows the parallel and perpendicular temperature ratios of $\alpha$ particles and protons for the case when the two species were initialized with equal thermal speeds. In this case, the parallel and perpendicular temperature curves do not overlap with each other and there is a general trend that the perpendicular heating of $\alpha$ s relative to protons gradually decreases with increasing relative drift, whereas the parallel ratio almost does not change. We should note that the parametric instabilities at high drifts make the system very unstable and hence we have not included data points with $V_{\alpha p}>0.5 V_{\mathrm{A}}$ in this plot. As we go to higher plasma $\tilde{\beta}$, the system becomes unstable at even lower initial drifts.

Figure 6 shows the parallel and perpendicular temperature ratios corresponding to two cases with a high plasma $\tilde{\beta}$. From this and the rest of the figures, one can see that the magnitude of the perpendicular temperature ratios decreases with increasing values of $\tilde{\beta}$. This is consistent with the decreasing temperature 
anisotropy for the $\alpha \mathrm{s}$ and its gradual increase for the protons as we move from low- to high- $\tilde{\beta}$ regimes. Furthermore, the preferential perpendicular heating of the minority $\alpha$ particles is most effective at low differential ion motion, but tends to become less efficient once they drift substantially faster than the majority protons carrying the waves.

The perpendicular ion temperature ratios $T_{\perp \alpha} / T_{\perp p}$ obtained from the numerical simulations are about twice as high as the ones derived based on Wind plasma measurements, which might be a model artifact caused by the large amplitude of the pump wave used in the simulations. If we reduce the wave amplitude to $\delta B / B_{0}=0.125$, the nonlinear effects are still present, but the resulting values of the ion temperature ratios fall within the observed range. Decreasing the wave amplitude, however, increases the time it takes for the parametric instabilities to develop, reduces the minor ion acceleration, and overall slows down and reduces the heating rate.

Figure 7 shows a scatter plot of the final ion temperature anisotropies versus the final plasma $\tilde{\beta}$. The results for the anisotropy $-\tilde{\beta}$ relation for the protons fall within the majority of data points, as actually observed in the fast solar wind (Bale et al. 2009; Gary et al. 2002). Yet, the simulation statistics are not sufficiently complete to check whether parametric instabilities can better explain the regulation of the ion temperature anisotropies in fast solar wind, which so far have been shown to be constrained essentially by the mirror and firehose instabilities.

Figure 8 shows the final normalized relative ion differential speed as a function of the final ion plasma $\tilde{\beta}$, separately for the two ion species. The relative drift is strongly dependent on the individual ion temperature and it is easier to preferentially accelerate the $\alpha$ particles at low values of the plasma $\tilde{\beta}$. At the same time, we may also interpret the information contained in that plot such that it is easier to heat the $\alpha$ particles in the lower relative drift regime up to $V_{\alpha p \text {, in }}=0.5 V_{\mathrm{A}}$. The opposite is true for the protons. With their lower gyrofrequency, the $\alpha$ particles are the first to extract energy from the waves at low relative drifts and it is easier to heat protons at high drifts, as the value of the differential streaming changes their kinematic conditions; see Equation (2). However, this deduction is valid only for small initial drifts with $V_{\alpha p \text {,in }} / V_{\mathrm{A}}$ below 0.5 . Above that value, the character of the parametric instabilities changes, the model plasma becomes quickly unstable (after a few proton gyroperiods), and an analog of the ion-beam magnetosonic instability occurs. It reduces the value of the initial relative drift and produces strong daughter IAWs, which are quickly absorbed via Landau damping and heat both ion species in the parallel direction. We should note that for the entire range of initial plasma parameters the system is, in principle, stable with respect to the linear magnetosonic instability, corresponding to

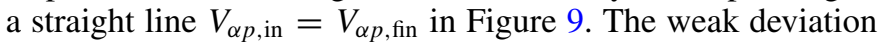
from the straight line, indicated by the open blue squares for the simulation case without pump waves, is due to the excitation of $\alpha$ /proton IAWs. Note that the initial drift values chosen along the line are below the linear theory thresholds conditions for $\alpha$ /proton electromagnetic instabilities. This result implies that wave-particle scattering by IAWs might help to constrain the values of $V_{\alpha p}$, even in the absence of transverse waves for some regions with low- $\beta$ conditions in the corona or the solar wind. When considered, however, the finite-amplitude Alfvén-cyclotron waves provoke effective nonlinear couplings via parametric decays, which strongly destabilize the system

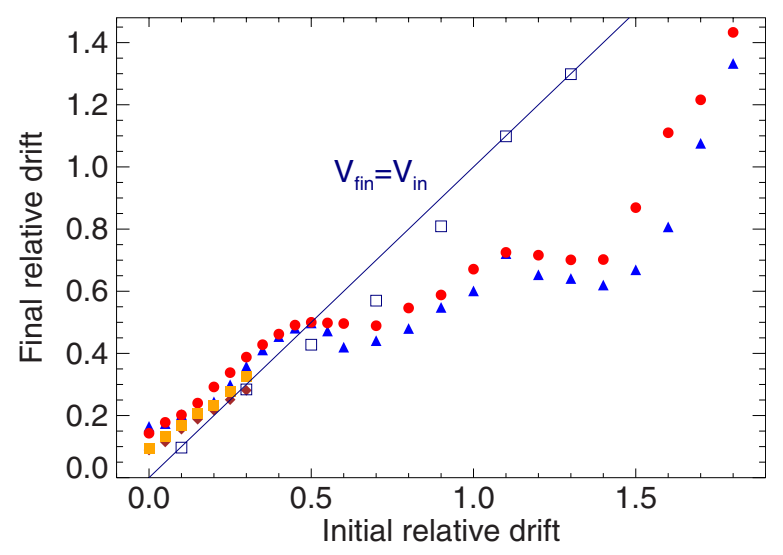

Figure 9. Final vs. initial relative drifts for different initial parallel plasma $\tilde{\beta}_{s}$ values. Brown rhombuses correspond to $\tilde{\beta}_{\alpha}=\tilde{\beta}_{p}=0.35$, case (d) and orange squares correspond to $\tilde{\beta}_{\alpha}=\tilde{\beta}_{p}=0.2$, case (c). Blue triangles denote case (b), with $\tilde{\beta}_{\alpha}=\tilde{\beta}_{p}=0.08$, and red circles stand for case (a), with $\tilde{\beta}_{\alpha}=0.02$ and $\tilde{\beta}_{p}=0.08$. Open blue squares correspond to simulations without the pump. The straight blue line denotes the linear theory predictions for the chosen set of plasma parameters.

(A color version of this figure is available in the online journal.)

once $V_{\alpha p, \text { in }}>0.5 V_{\mathrm{A}}$ and provide an additional source for parallel heating.

In other words, this means that nonlinear Landau damping and pitch-angle scattering are most effective for the $\alpha$ particles at low relative drifts. We should note that this result is limited by the initial conditions like the pump wave number, although recent observations detect maximum wave activity at similar wave numbers and wave frequencies (Jian et al. 2009), which favors our heating scenario based on nonlinear waves. The effects of a broadband initial spectrum of nonlinear waves are discussed in Maneva et al. (2013b).

Figure 9 shows the change of the initial relative drift speed for the four investigated initial plasma $\tilde{\beta}$ conditions. The figure demonstrates that the waves cannot accelerate the ions to relative velocities higher than $V_{\alpha p \text {,in }}=0.5 V_{\mathrm{A}}$, even in low- $\tilde{\beta}$ regimes. Above this value, the instabilities mark a strong transition and a reduction of the initial drift is observed. Thus, even though it can stabilize the ion-beam instability (Gomberoff 2003), the role of the pump wave in this case is to destabilize the system via a parametric instability analogous to the ionbeam magnetosonic instability. Linear Vlasov theory for a zeropump wave indicates that the plasma system should be stable with respect to electromagnetic excitations for all values of the initial drifts used in our simulations, so that there should be no change in the relative drift during each simulation run. Thus, in the absence of waves, the data in Figure 9 should follow a straight line. Indeed, simulations without a pump wave show slight reductions of the drift speed around the critical value $V_{\alpha p \text {,in }}=0.5 V_{\mathrm{A}}$. This is an indication that IAWs can be easily excited in such regimes. Although not presented here, this has been confirmed by the parametric instability theory. For some examples of parametric instabilities in a drifting proton- $\alpha$ kinetic plasma, see Gao et al. (2013), Araneda et al. (2009), and Maneva et al. (2009). For the case of proton- $\alpha$-oxygen plasma, see Maneva et al. (2010). A recent comparison between the theoretically predicted growth rates for daughter IAWs in a proton- $\alpha$ plasma and the growth rates of the ion density fluctuations calculated during the linear stage of hybrid simulations can be found in Maneva et al. (2013a). 


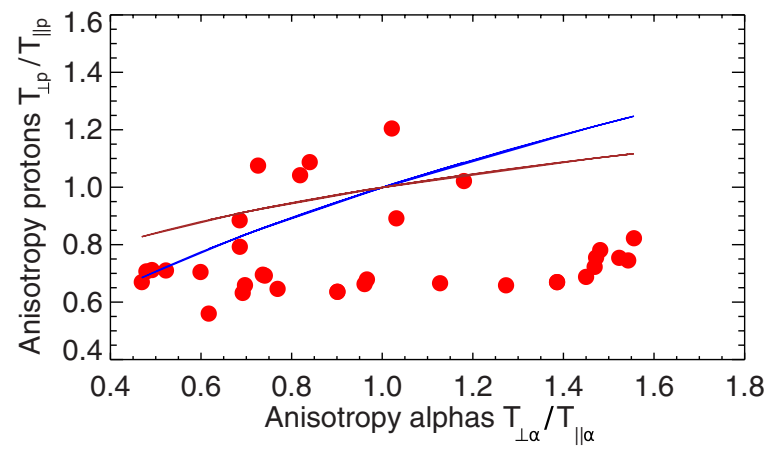

Figure 10. Scatter plot of the relation between the ion temperature anisotropy for protons and $\alpha$ particles calculated at the final stage of the simulations for case (a) with $\tilde{\beta}_{\alpha}=0.02$ and $\tilde{\beta}_{p}=0.08$. Overplotted are two curves of the type $A_{\alpha}=A_{p}^{s}$, with $s=0.5$ given in blue and $s=0.25$ represented by the brown line.

(A color version of this figure is available in the online journal.)

We should note that the nature of the parametric instabilities that occur throughout the simulations presented here (whether decay, beam, or modulational) and the phase speeds of the generated daughter waves strongly depend on the initial plasma parameters. Since we do not vary the minor ion density and consider initially isotropic distributions for all cases, the instabilities are mainly affected by the value of the relative drift speed and the ion plasma $\tilde{\beta}$. It is beyond the scope of this paper to demonstrate the kinetics of the ion trapping causing beam formations and other relevant wave-particle interactions with the daughter waves. Such an analysis has been performed and detailed results of the generated wave spectra and their implications for the ion heating, trapping, and acceleration/deceleration will be presented elsewhere.

Since strong IAWs (with phase speeds $0.4-0.7 V_{\mathrm{A}}$ ) are easily excited in the low relative drift speed regime, the pump can accelerate the $\alpha$ particles to higher values of the relative drift (for $V_{\alpha p \text {,in }}<0.5 V_{\mathrm{A}}$ ) or significantly slow them down (for $V_{\alpha p \text {,in }} \geqslant 0.6 V_{\mathrm{A}}$ ). In addition, for $V_{\alpha p \text {,in }} \geqslant 0.9 V_{\mathrm{A}}$, when $V_{\alpha p}>\omega_{0} / k_{0}$ (see Table 1 ), there is a short initial phase of about $300 \Omega_{p}{ }^{-1}$ where the differential streaming of the $\alpha$ particles remains almost constant, after which it starts to decrease. For that value of the initial relative drift, the phase speed of the pump is closest to the bulk velocity of the $\alpha$ particles, so that for a short time the $\alpha$ s are "surfing" the waves.

In contrast, at low drifts $\left(V_{\alpha p \text {, in }}<0.5 V_{\mathrm{A}}\right)$, the resonant daughter IAWs have phase speeds larger than the bulk speed of the $\alpha$ particles and thus the latter get accelerated. Additionally, the pump in those cases can cause many more oscillations in the bulk moments (including the parallel motion) of the $\alpha$ particles and less in the bulk moments of the protons, whereas at high drifts the opposite effect is observed. Finally, we should note that for all data points with initial relative drifts between $0.5 V_{\mathrm{A}}$ and $1.4 V_{\mathrm{A}}$, the simulations do not reach a stationary state and hence we have included them here only to illustrate the vital effects of the parametric instabilities.

Figure 10 shows the relation of the temperature anisotropies between the two ion species for case (a). The plot exhibits very similar features to the results from the recent data analysis of in situ solar wind measurements taken by the Faraday cups onboard the Wind spacecraft; see Figure 1 in Maruca et al. (2012). Similar results have recently been reported from 2D hybrid Vlasov-Maxwell simulations; see Figure 7 in Perrone et al. (2013). However, their model results produce a very different relation between the temperature anisotropy of the $\alpha$ particles and the relative drift speed; see Figure 8 in the same paper.

\section{CONCLUSIONS}

The results from the series of $1.5 \mathrm{D}$ hybrid simulations presented here provide new insights into the generation and regulation of the proton and $\alpha$ particle thermal anisotropies, as well as the ion differential streaming in a dilute and collisionless plasma prevailing in coronal holes and the associated fast solar wind streams. The analysis shows that the proposed scenario for preferential and anisotropic heating and acceleration by parametric decay of a large-amplitude Alfvén-cyclotron pump wave is able to reproduce key features of the behavior of nonthermal ions, as measured in situ in the solar wind.

The current simulation model is applicable to other space plasma environments, wherever we have the required $\tilde{\beta}$ conditions and a collisionless plasma with homogeneous magnetic background and superimposed high-frequency nonlinear fluctuations. Yet, the scenario considered here is still far from representing a self-consistent model of the real conditions in the fast solar wind, as we imposed a single monochromatic Alfvéncyclotron wave and we treated the plasma as homogeneous. Also, no gravity effects were taken into account, nor were the solar wind expansion and the related change of the Alfvén speed properly handled. Even so, we are able to capture some main characteristics of the non-thermal ions, streaming differentially in the fast solar wind. We derived the parallel heating of $\alpha$ particles at high relative drifts and provided a possible explanation for the more than mass-proportional preferential heating and differential acceleration of minor ions, represented by the $\alpha$ particles here. An attempt to improve the current model including broadband, turbulent wave spectra within the expanding box is presented in Maneva et al. (2013b).

The resulting relative drift speeds and ion temperature anisotropies lay within the range observed by Helios (Marsch 2006; Bourouaine et al. 2011) and Ulysses (Neugebauer et al. 1996) and their relation also is in good agreement with recent observations made by the Wind spacecraft in the fast solar wind near the Earth (Kasper et al. 2008, 2013; Maruca et al. 2011, 2012). The maximal differential speed reached in the model is $V_{\alpha p}=0.5 V_{\mathrm{A}}$. For all cases when $V_{\alpha p}<0.5 V_{\mathrm{A}}$, the simulations show more than mass-proportional heating, giving $T_{\alpha}>4 T_{p}$, in good agreement with the in situ measurements of fast solar wind plasma made by the Wind and Ulysses spacecrafts (Cohen et al. 1996). The obtained parallel and perpendicular $\alpha$-to-proton temperature ratios, $T_{\| \alpha} / T_{\| p}, T_{\perp \alpha} / T_{\perp p}$, in our model, however, seem to be enhanced by up to a factor of two. This may be attributed to the insufficiency of the initial simulation setup, assuming a rather large-amplitude pure monochromatic pump wave or different thermal speeds at the beginning of the simulations. A decrease of the initial pump wave amplitude by a factor of two brings the temperature ratios to within the observed values.

The simulations clearly indicate that it seems difficult to form and maintain highly anisotropic drifting ion velocity distributions if the ions are heated only locally in high- $\tilde{\beta}$ conditions and that therefore the observed anisotropic ions with high relative drifts have possibly been heated earlier, in the low$\tilde{\beta}$ regions closer to the Sun. If the particles interacted there with high-frequency, large-amplitude waves, they might be able to acquire significant relative drifts and develop anisotropic 
distributions, which can be stabilized by wave-particle interactions mediated by wave activity that is generated through parametric instabilities.

Thus, while initially drifting only at some fraction of the large local Alfvén speed in a coronal hole, for instance, the ions could acquire increasingly larger normalized relative drifts $V_{\alpha p} / V_{\mathrm{A}}$ as they travel farther away from the Sun where the Alfvén speed naturally decreases together with the diminishing solar magnetic field. This would continue until the particles cross a marginal stability region of a linear plasma instability (like the firehose and the mirror) or can excite a parametric instability depending to the concomitant background wave spectra.

The results presented in this paper primarily address finiteamplitude, highly nonlinear monochromatic waves (with amplitudes that constitute $25 \%$ of the background magnetic field magnitude). However, our complementary studies with lower wave amplitudes and with initial broadband wave spectra (Maneva et al. 2013b) confirm that lower amplitude waves from turbulent wave spectra could also be used for proton beam formations, preferential heating, and differential acceleration for the minor ions (as shown for the case of $\alpha$ particles). This seems to be a steady effect observed in $1.5 \mathrm{D}$, as well as in $2.5 \mathrm{D}$ hybrid simulations. Furthermore, the amount of heating and differential streaming acquired by the waves depends strongly on the initial wave amplitudes, as well as on their frequency range and on the angle of propagation (see Y. G. Maneva et al. 2014, in preparation). Maneva et al. (2013b) and Y. G. Maneva et al. (2014, in preparation) also address the effect of the solar wind expansion, which cools the ions in the perpendicular direction, affects the differential streaming, and influences the turbulent cascade, bringing wave modes in or out of resonance with the particles.

This work was initiated as part of a $\mathrm{PhD}$ thesis at the University of Göttingen and funded within the framework of the International Max Planck Research School (IMPRS) by the MPS in Katlenburg-Lindau, Germany. The research was finalized at the University of Concepción and supported in part by FONDECYT grant No. 1110880. Partial support for this paper came from NASA grant NNX10AC56G.

\section{REFERENCES}

Abraham-Shrauner, B., \& Feldman, W. C. 1977, JGR, 82, 618 Araneda, J. A., Maneva, Y., \& Marsch, E. 2009, PhRvL, 102, 175001 Bale, S. D., Kasper, J. C., Howes, G. G., et al. 2009, PhRvL, 103, 211101 Banerjee, D., Pérez-Suárez, D., \& Doyle, J. G. 2009, A\&A, 501, L15
Berger, L., Wimmer-Schweingruber, R. F., \& Gloeckler, G. 2010, in AIP Conf. Proc. 1216, Twelfth International Solar Wind Conference, ed. M. Maksimovic, N. Meyer-Vernet, M. Moncuquet, \& F. Pantellini (Melville, NY: AIP), 253

Berger, L., Wimmer-Schweingruber, R. F., \& Gloeckler, G. 2011, PhRvL, 106, 151103

Bourouaine, S., Marsch, E., \& Neubauer, F. M. 2010, GeoRL, 37, 14104

Bourouaine, S., Marsch, E., \& Neubauer, F. M. 2011, ApJL, 728, L3

Bruno, R., \& Carbone, V. 2005, LRSP, 2, 4

Cohen, C. M. S., Collier, M. R., Hamilton, D. C., et al. 1996, GeoRL, 23, 1187

Galinsky, V. L., \& Shevchenko, V. I. 2012, ApJ, 751, 146

Galinsky, V. L., \& Shevchenko, V. I. 2013, ApJ, 763, 31

Gao, X., Lu, Q., Tao, X., Hao, Y., \& Wang, S. 2013, PhPl, 20, 092106

Gary, S. P., Goldstein, B. E., \& Neugebauer, M. 2002, JGRA, 107, 1169

Gary, S. P., Yin, L., \& Winske, D. 2006, JGR, 111, 6105

Gomberoff, L. 2003, JGRA, 108, 1261

Hellinger, P., Velli, M., Trávníček, P., et al. 2005, JGR, 110, 12109

Hollweg, J. V., Esser, R., \& Jayanti, V. 1993, JGR, 98, 3491

Hollweg, J. V., \& Isenberg, P. A. 2002, JGR, 107, 12-1

Jess, D. B., Mathioudakis, M., Erdélyi, R., et al. 2009, Sci, 323, 1582

Jian, L. K., Russell, C. T., Luhmann, J. G., et al. 2009, ApJL, 701, L105

Jian, L. K., Russell, C. T., Luhmann, J. G., et al. 2010, JGR, 115, 12115

Kasper, J. C., Lazarus, A. J., \& Gary, S. P. 2008, PhRvL, 101, 261103

Kasper, J. C., Maruca, B. A., Stevens, M. L., \& Zaslavsky, A. 2013, PhRvL, 110,091102

Kauffmann, K., \& Araneda, J. A. 2008, PhPl, 15, 062106

Kohl, J. L., Noci, G., Cranmer, S. R., \& Raymond, J. C. 2006, A\&ARv, 13, 31

Maneva, Y. G., Araneda, J. A., \& Marsch, E. 2009, in AIP Conf. Proc. 1121, Space Plasma Physics, ed. I. Zhelyazkov (Melville, NY: AIP), 122

Maneva, Y. G., Araneda, J. A., \& Marsch, E. 2010, in AIP Conf. Proc. 1216, Twelfth International Solar Wind Conference, ed. M. Maksimovic, N. MeyerVernet, M. Moncuquet, \& F. Pantellini (Melville, NY: AIP), 227

Maneva, Y. G., Ofman, L., \& Viñas, A. F. 2013a, in AIP Conf. Proc. 1539, Solar Wind 13, ed. G. P. Zank, J. Borovsky, R. Bruno, J. Cirtain, S. Cranmer, H. Elliott, J. Giacalone, W. Gonzalez, G. Li, E. Marsch, E. Moebius, N. Pogorelov, J. Spann, \& O. Verkhoglyadova (Melville, NY: AIP), 34

Maneva, Y. G., Viñas, A. F., \& Ofman, L. 2013b, JGRA, 118, 2842

Marsch, E. 2006, LRSP, 3, 1

Marsch, E., Rosenbauer, H., Schwenn, R., Muehlhaeuser, K., \& Neubauer, F. M. 1982a, JGR, 87, 35

Marsch, E., Schwenn, R., Rosenbauer, H., et al. 1982b, JGR, 87, 52

Maruca, B. A., Kasper, J. C., \& Bale, S. D. 2011, PhRvL, 107, 201101

Maruca, B. A., Kasper, J. C., \& Gary, S. P. 2012, ApJ, 748, 137

Matteini, L., Hellinger, P., Goldstein, B. E., et al. 2013, JGR, 118, 2771

Matteini, L., Landi, S., Hellinger, P., et al. 2007, GeoRL, 34, 20105

Matteini, L., Landi, S., Velli, M., \& Hellinger, P. 2010, JGR, 115, 9106

Nariyuki, Y., Hada, T., \& Tsubouchi, K. 2009, JGR, 114, 7102

Neugebauer, M., Goldstein, B. E., Smith, E. J., \& Feldman, W. C. 1996, JGR, 101, 17047

Perrone, D., Valentini, F., Servidio, S., Dalena, S., \& Veltri, P. 2013, ApJ, 762, 99

Sonnerup, B. U. Ö., \& Su, S.-Y. 1967, PhFl, 10, 462

Terasawa, T., Hoshino, M., Sakai, J., \& Hada, T. 1986, JGR, 91, 4171

Tomczyk, S., McIntosh, S. W., Keil, S. L., et al. 2007, Sci, 317, 1192

Tu, C.-Y., \& Marsch, E. 1995, SSRv, 73, 1

Verscharen, D., \& Chandran, B. D. G. 2013, ApJ, 764, 88

Winske, D., \& Leroy, M. M. 1984, JGR, 89, 2673 